\title{
Divergência genética de Castanheira-do-Brasil por meio de caracteres biométricos de frutos e sementes
}

A Castanheira-do-brasil é considera uma das espécies mais importantes da Floresta Amazônica, por contribuir para a subsistência de muitas comunidades locais. Objetivou-se determinar a divergência em Castanheira-do-Brasil, com propósito de subsidiar trabalhos de seleção de matrizes para coleta de sementes, programas de conservação e melhoramento genético da espécie. Foram avaliados 210 frutos e 2100 sementes coletados em 21 matrizes em Itaúba (MT) na safra $2016 / 2017$. Os caracteres: massa total do fruto (MTF, g), diâmetro longitudinal do fruto (DLF, mm), diâmetro transversal do fruto (DTF, mm), espessura da casca do fruto (ECF, $\mathrm{mm}$ ), número de sementes por fruto (NSF), massa fresca das sementes (MFS, g), massa seca das sementes (MSS, g), massa unitária da semente (MUS, g), maior eixo da semente (MAS, $\mathrm{mm}$ ), eixo médio da semente (EMS, $\mathrm{mm}$ ), menor eixo da semente (MES, $\mathrm{mm}$ ) e massa unitária da amêndoa (MUA, $\mathrm{g}$ ) foram avaliados. Análises multivariadas foram realizadas para a obtenção das estimativas de divergência genética por meio do método da distância Euclidiana Média Padronizada. Com base na matriz de distância gerada foi realizado o agrupamento das matrizes. As distâncias entre os pares de matrizes variaram de 0,11 a 0,84 , com média de 0,32. A maior divergência dii' = 0,84 foi encontrada entre as matrizes ITA19 e ITA5. Os caracteres de maior contribuição para discriminação das matrizes foi massa fresca das sementes, massa seca das sementes, massa unitária da semente e maior eixo da semente. Há divergência genética entre as árvores matrizes de Castanheira-do-Brasil, podendo ser utilizadas para a produção de mudas via coleta de sementes ou seleção de gemas para bancos de germoplasma e para produtores que desejam reflorestar áreas degradas ou realizar plantios comerciais.

Palavras-chave: Bertholletia excelsa; Conservação; Recursos Genéticos.

\section{Genetic divergence of Brazil nut through biometric fruit and seed characters}

\begin{abstract}
Brazil nut tree is considered one of the most important species of the Amazon rainforest, as it contributes to the subsistence of many local communities. The objective of this study was to determine the divergence in Castanheira-do-Brasil, with the purpose of subsidizing seed selection work for seed collection, conservation programs and genetic improvement of the species. Were evaluated 210 fruits and 2100 seeds collected in 21 matrices in Itaúba (MT) in the $2016 / 2017$ crop. The characters: total fruit mass (MTF, g), longitudinal fruit diameter (DLF, mm), transversal fruit diameter (DTF, mm), fruit peel thickness (ECF, mm), number of seeds per fruit ( NSF), fresh seed mass (MFS, g), dry seed mass (MSS, g), unit seed mass (MUS, g), largest seed axis (MAS, mm), average seed axis (EMS, mm), smaller seed axis (MES, mm) and almond unit mass (MUA, g) were evaluated. Multivariate analyzes were performed to obtain the genetic divergence estimates using the Standardized Euclidean Distance Method. Based on the generated distance matrix, the matrix grouping was performed. The distances between the pairs of matrices ranged from 0.11 to 0.84 , with an average of 0.32 . The greatest divergence dii ' $=0.84$ was found between matrices ITA19 and ITA5. The characters that most contributed to the discrimination of the matrices were seed fresh mass, seed dry mass, seed unit mass and major seed axis. There is genetic divergence among the Castanheira-do-Brasil mother trees, which can be used for seedling production via seed collection or bud selection for germplasm banks and for producers who wish to reforest degraded areas or to make commercial plantations.
\end{abstract}

Keywords: Bertholletia excelsa; Conservation; Genetic Resources.

Topic: Uso dos Recursos Naturais

Reviewed anonymously in the process of blind peer.
Received: 13/10/2018

Approved: 13/11/2018
Bruno Vindilino Roelis (D)

Universidade do Estado de Mato Grosso, Brasil

http://lattes.cnpq.br/7085391393114086

http://orcid.org/0000-0002-4335-5894

brunovindilino@gmail.com

Ana Aparecida Bandini Rossi (iD)

Universidade Federal da Paraíba, Brasil

http://lattes.cnpq.br/2734433144153549

http://orcid.org/0000-0002-8318-5375

anabanrossi@gmail.com

\section{Aisy Botega Baldoni (D)}

Universidade de Brasília, Brasil

http://lattes.cnpq.br/7489953068553839

http://orcid.org/0000-0002-8355-6957

aisybb@gmail.com

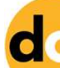

DOI: 10.6008/CBPC2179-6858.2018.008.0022

\author{
Auana Vicente Tiago \\ Universidade Federal da Paraíba, Brasil \\ http://lattes.cnpq.br/3112795576497501 \\ http://orcid.org/0000-0001-9556-9491 \\ auana bio@hotmail.com \\ Helio Tonini (it) \\ Universidade Federal de Santa Maria, Brasil \\ http://lattes.cnpq.br/9255737430748882 \\ http://orcid.org/0000-0003-1123-7604 \\ helio.tonini@embrapa.br
}

\section{Referencing this:}

ROELIS, B. V.; ROSSI, A. A. B.; BALDONI, A. B.; TIAGO, A. V.; TONINI, H.. Divergência genética de Castanheira-do-Brasil por meio de caracteres biométricos de frutos e sementes. Revista Ibero-Americana de Ciências Ambientais, v.9, n.8, p.249-259, 2018. DOI:

http://doi.org/10.6008/CBPC2179-6858.2018.008.0022 


\section{INTRODUÇÃO}

A Castanheira-do-Brasil (Bertholletia excelsa Bonpl., Lechytidaceae) é uma das árvores-símbolo da Amazônia, devido a sua importância social, ecológica e econômica para a região. É popularmente conhecida como castanha-do-pará ou castanha-do-brasil e, mais recentemente, castanha-da-Amazônia (WADT et al., 2012). As sementes comestíveis da castanheira são de grande importância para as comunidades locais por serem uma das principais fontes de renda, especialmente durante o período chuvoso do ano (CLAY, 1997; ORTIZ, 2002).

A produção da castanha é obtida quase exclusivamente de atividade extrativa florestal, sendo as plantações pouco significativas em termos quantitativos (ZUIDEMA, 2003). Trata-se de um produto de importância econômica internacional com um papel importante na conservação e no desenvolvimento socioeconômico da região. Em termos comerciais, a maior parte das sementes é vendida para o mercado nacional e internacional, poucas são comercializadas em nível local ou regional (MORI et al., 1990; CLAY, 1997; CLEMENT, 1999).

A exploração da castanha-do-brasil não tem se fundamentado, tradicionalmente, no entendimento ecológico da dinâmica da população da espécie, tão pouco na variabilidade natural que existe procurando características morfológicas e genéticas que possam auxiliar na decisão sobre técnicas de manejo como o plantio de enriquecimento, a seleção de árvores mais produtivas, entre outras (BRAGA, 2007).

Estudos biométricos auxiliam a determinação de diferentes parâmetros fenotípicos entre os indivíduos de uma população, visto que, nas espécies arbóreas tropicais existe grande variabilidade com relação aos caracteres morfológicos (CRUZ et al., 2003; MELO et al., 2004). Dessa forma, é de grande importância o conhecimento do tipo e tamanho de frutos e sementes para esses estudos (CRUZ et al., 2001). Entretanto, poucos são os trabalhos que objetivam a caracterização de frutos e sementes de espécies arbóreas visando ampliar o conhecimento sobre elas (EDWARDS, 2000).

Dentro da mesma espécie, existem variações individuais devido às influências de fatores bióticos e abióticos, durante o desenvolvimento das sementes e à variabilidade genética. Assim, o tamanho e o peso da semente podem variar entre plantas da mesma espécie, de ano para ano e, também, dentro de uma mesma planta (PIÑA-RODRIGUES et al., 1993). A variação entre indivíduos numa mesma população possibilita a seleção com vistas à melhoria de um dado caráter, constituindo-se numa das mais importantes fontes de variabilidade disponíveis para os melhoristas de plantas (PAULA, 2007).

Levando em consideração que a castanha-do-brasil é um dos produtos não madeireiros mais importantes da economia florestal da Amazônia o objetivo deste estudo foi determinar a divergência genética por caracteres biométricos de frutos e sementes, entre matrizes de castanheira-do-brasil, com propósito de subsidiar trabalhos de seleção de matrizes para coleta de sementes, programas de conservação e melhoramento genético da espécie. 


\section{MATERIAIS E MÉTODOS}

\section{Local de Coleta dos Frutos}

Os frutos de Castanheira-do-Brasil foram coletados 21 matrizes selecionadas na reserva legal da Fazenda Santo Ângelo, localizada no município de Itaúba, situado na região norte do estado de Mato Grosso,

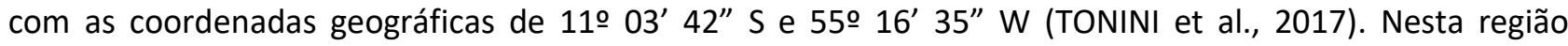
predomina habitats antropizados, derivado a expansão da fronteira agrícola. Foram coletados dez frutos maduros em cada uma das 21 matrizes na safra 2016/2017, totalizando 210 frutos. Os materiais coletados foram devidamente identificados em campo e transportados para a Embrapa Agrossilvipastoril, Sinop (MT), onde foram avaliados.

\section{Características biométricas do fruto}

Os frutos foram avaliados por meio das características: Massa total do fruto (MTF) (g): medida em balança de precisão de 0,001g. Diâmetro longitudinal do fruto (DLF) (mm): dado pela distância entre a base e o ápice. Medida a circunferência por meio de uma fita métrica e em seguida os dados foram transformados em diâmetro dividindo a circunferência por $\pi$ (pi). Tem-se DLF= circunferência longitudunal do fruto

Diâmetro transversal do fruto (DTF) (mm): dado pela maior medida ortogonal (linha reta paralela) ao comprimento. Avaliada a circunferência por meio de uma fita métrica e em seguida os dados foram transformados em diâmetro dividindo a circunferência por $\pi$ (pi). Tem-se, também, DTF = $\frac{\text { circunferência transversal do fruto }}{\pi}$. Espessura da casca do fruto $(E C F)(\mathrm{cm})$ : média de três avaliações realizadas, com paquímetro digital, em locais diferentes da casca do fruto.

Foram avaliados também o número de sementes por fruto (NSF), a massa fresca das sementes (MFS) (g) e a massa seca das sementes (MSS) (g) em uma balança de precisão de 0,001g. A massa seca das sementes foi obtida através de secagem em estufa com temperatura média de $60^{\circ} \mathrm{C}$ até que a massa permanecesse constante.

\section{Características biométricas das sementes secas}

Foram selecionadas aleatoriamente dez sementes por fruto para avaliação, totalizando 2.100 sementes. As sementes foram avaliadas por meio das seguintes características: Massa unitária da semente (MUS) (g): medida por meio de uma balança semianalítica, capacidade de $620 \mathrm{~g}$ com divisão de $0,001 \mathrm{~g}$. Maior eixo da semente (MAS) (mm): é a medida da maior dimensão, avaliada por meio de um paquímetro digital.

Eixo médio da semente (EMS) $(\mathrm{mm})$ : medida de forma vertical com a semente deitada, avaliada por meio de um paquímetro digital. Menor eixo da semente (MES) (mm): medida de forma horizontal em relação a base da semente, avaliada por meio de um paquímetro digital. Massa unitária da amêndoa (MUA) (g): medida em uma balança semianalítica $620 \mathrm{~g}$ com divisão de $0,001 \mathrm{~g}$. 


\section{Análise estatística}

Os dados obtidos foram inicialmente submetidos à análise estatística descritiva utilizando medidas de tendência central (média), de variabilidade de dados (desvio padrão) e a diferença entre o maior e o menor valor do conjunto de dados. Todas as análises foram realizadas utilizando o aplicativo computacional em genética e estatística 'Programa GENES' (CRUZ, 2006).

Foram realizadas análises multivariadas para a obtenção das estimativas de divergência genética das matrizes por meio do método da distância Euclidiana Média Padronizada. A matriz de dissimilaridade gerada foi utilizada para a realização da análise de agrupamento pelos métodos UPGMA (SNEATH et al., 1973) e pelo método de otimização de Tocher (RAO, 1952). A validação do agrupamento pelo método UPGMA foi determinada pelo coeficiente de correlação cofenético (CCC) (SOKAL et al., 1962).

A análise de componentes principais $(A C P)$ foi realizada a fim de definir as características de maior contribuição para o estudo de diversidade. A técnica de componentes principais, a partir da matriz de correlação, consiste em transformar um conjunto de $p$ variáveis $X 1, X 2, \ldots$; Xp em um novo conjunto $Y 1$, $\mathrm{Y} 2, \ldots, \mathrm{Yp}$, em que os $\mathrm{Y}^{\prime} \mathrm{s}$ apresentam as seguintes propriedades: Cada componente principal (Yi) é uma combinação linear das doze variáveis padronizadas $(\mathrm{Xj})$, ou seja, $Y_{i}=a_{i 1} X_{1}+a_{i 2} X_{2}+\ldots+a_{i p} X_{p}=$ $\sum_{j=1}^{p} a_{i j} X_{j}$, onde $a_{\mathrm{ij}}$ são os autovetores, com $\mathrm{i}=1,2, \ldots . . \mathrm{p}$ e $\sum_{j=1}^{p} a_{i j}^{2}=1$; o primeiro componente principal, $Y_{1}$, é tal que sua variância é máxima entre todas as combinações lineares de $X$. O segundo componente principal é não correlacionado com o primeiro e possui a segunda maior variância.

Da mesma forma, definam-se os outros p componentes principais não-correlacionados entre si, ou seja, $\operatorname{Var}\left(\mathrm{Y}_{1}\right) \geq \operatorname{Var}\left(\mathrm{Y}_{2}\right) \geq \ldots \geq \operatorname{Var}\left(\mathrm{Y}_{\mathrm{p}}\right)$; A cada componente principal $\mathrm{Y}_{\mathrm{i}}$ existe $p$ autovalor ordenado de forma que se obtenha $\lambda_{1} \geq \lambda_{2} \geq \ldots \geq \lambda_{p}$; As combinações lineares formadas são não-correlacionadas: $\operatorname{Cov}\left(Y_{1}, Y_{2}\right)=\operatorname{Cov}\left(Y_{1}, Y_{3}\right)=\ldots=\operatorname{Cov}\left(Y_{p}-1, Y_{p}\right)=0 ;$ A importância relativa de um componente principal foi avaliada pela percentagem de variância total que ele explica, ou seja, a percentagem de seu autovalor em relação ao total dos autovalores de todos os componentes que é dado pela equação $Y_{i}=\frac{\operatorname{Var}\left(Y_{i}\right)}{\sum_{i=1}^{p} \operatorname{Var}\left(Y_{i}\right)} x 100=$ $\frac{\lambda_{i}}{\sum_{i=1}^{p} \lambda_{i}} x 100$. Para todas as análises estatísticas foi utilizado o programa computacional GENES (CRUZ, 2006).

\section{RESULTADOS E DISCUSSÃO}

A análise descritiva revelou a existência de divergência genética entre os 21 genótipos de castanheira-do-brasil com base nas características de frutos e sementes avaliados. Ocorreu variação para as variáveis, o que pode ser verificado pela magnitude dos valores de amplitude (tabela 1). Em relação ao diâmetro transversal do fruto, os valores obtidos neste estudo, que variou de 79,62mm a 125,48mm.

A partir disso, teve-se uma média de 99,79mm foram menores em relação aos observados por Kaminski et al. (2008), que avaliou frutos de castanheira-do-brasil oriundos de duas parcelas permanentes de floresta nativa do Projeto Kamukaia nos municípios de Caracaí e São João da Baliza, no sul do Estado de Roraima e encontrou uma média de $118,91 \mathrm{~mm}$, com valores variando de $91,26 \mathrm{~mm}$ a $138,21 \mathrm{~mm}$. A média de 
diâmetro longitudinal encontrada por Kamisnki et al. (2008) também se mostrou maior em relação ao valor encontrado neste trabalho, com o valor de $113,54 \mathrm{~mm}$ de comprimento.

Tabela 1: Análise descritiva apresentando as médias gerais, desvio-padrão (DP), valores de mínimo, máximo e amplitude das características avaliadas nas 21 matrizes de Castanheira-do-Brasil.

\begin{tabular}{|l|r|r|r|r|r|}
\hline Variáveis & \multicolumn{1}{|c|}{ Média } & \multicolumn{1}{|c|}{ DP } & \multicolumn{1}{|c|}{ Mínimo } & \multicolumn{1}{c|}{ Máximo } & \multicolumn{1}{c|}{ Amplitude } \\
\hline MTF (g) & 404,09 & 124,40 & 112 & 828 & 716 \\
\hline DLF $(\mathbf{m m})$ & 101,55 & 8,78 & 80,25 & 124,84 & 44,59 \\
\hline DTF $(\mathbf{m m})$ & 99,79 & 9,32 & 79,62 & 125,48 & 45,86 \\
\hline ECF $(\mathbf{m m})$ & 9,64 & 1,32 & 13,5 & 26 & 7,78 \\
\hline NSF & 16,48 & 3,70 & 24 & 182 & 16 \\
\hline MFS (g) & 94,54 & 27,49 & 20 & 120 & 158 \\
\hline MSS (g) & 65,75 & 19,66 & 10 & 100 \\
\hline
\end{tabular}

Legenda: MTF: massa total do fruto (g), DLF: Diâmetro longitudinal do fruto (mm), DTF: Diâmetro transversal do fruto $(\mathrm{mm})$, ECF: espessura da casca do fruto $(\mathrm{mm}), \mathrm{NSF}$ : número de sementes por fruto, MFS: massa fresca das sementes (g), MSS: massa seca das sementes (g).

A média da massa do fruto foi de 404,09g, sendo menor que as encontradas por Camargo et al. (2010) que ao analisarem três variações de castanheira-do-brasil (rajada, mirim e rosa) na Fazenda São Nicolau, Cotriguaçu (MT), encontraram as médias 481,37g, 434,35g e 697,13g para os tipos citados anteriormente. Com relação à massa das sementes por fruto, cujos valores médios foram de 94,54g para o massa fresca e 65,75g para a massa seca, os valores encontrados por Kaminski et al. (2008) e Camargo (2010), que trabalharam com a massa fresca das sementes, também foram maiores, sendo respectivamente 184,66g e 119,93g, 118,66g e 124,5g para os três tipos avaliados por Camargo et al. (2010).

Quanto ao número total de sementes, este estudo apresentou uma média de 16,48 , sendo semelhante ao valor encontrado por Kaminski et al. (2008) de 16,28, bem como para duas das variedades estudadas por Camargo et al. (2010), 16,51 (rajada) e 15,17 (mirim). As sementes apresentaram médias de 4,08g para a massa unitária da semente, $34,83 \mathrm{~mm}$ para o maior eixo, $20,52 \mathrm{~mm}$ para o eixo médio, $14,72 \mathrm{~mm}$ para o menor eixo e 1,79g para a massa unitária da amêndoa (tabela 2).

Tabela 2: Análise descritiva apresentando as médias gerais, desvio-padrão (DP), valores de mínimo, máximo e amplitude das características avaliadas nas 21 matrizes de Castanheira-do-Brasil.

\begin{tabular}{|l|r|r|r|r|r|}
\hline Variáveis & \multicolumn{1}{|c|}{ Média } & \multicolumn{1}{c|}{ DP } & \multicolumn{1}{c|}{ Mínimo } & \multicolumn{1}{c|}{ Máximo } & \multicolumn{1}{c|}{ Amplitude } \\
\hline MUS (g) & 4,08 & 0,97 & 1,33 & 6,68 & 5,35 \\
\hline MAS (mm) & 34,83 & 3,05 & 26,23 & 42,49 & 16,26 \\
\hline EMS (mm) & 20,52 & 1,63 & 16,29 & 26,22 & 9,93 \\
\hline MES (mm) & 14,72 & 1,31 & 10,73 & 18,32 & 7,59 \\
\hline MUA (g) & 1,79 & 0,53 & 0,28 & 3,28 & 3 \\
\hline
\end{tabular}

Legenda: MUF: massa unitária do fruto $(\mathrm{g}), \mathrm{MAS}$ : maior eixo da semente $(\mathrm{mm})$, EMS: eixo médio da semente $(\mathrm{mm})$, MES: menor eixo da semente (mm), MUA: massa unitária da amêndoa (g).

A grande amplitude observada entre os valores mínimos e máximos dos caracteres demonstra presença de variabilidade entre os genótipos avaliados, o que está de acordo com estudo realizado por Teixeira et al. (2015), o qual observou elevado nível de variação genética dentro de populações de castanheira, resultado da alta taxa de cruzamento entre os indivíduos (O'MALLEY et al., 1988).

Taffarel et al. (2013) e Nogueira (2014), ao analisarem sementes da mesma floresta nativa do município de Itaúba, encontraram médias maiores para o eixo médio $(24,19 \mathrm{~mm}$ e $26,60 \mathrm{~mm})$ e o menor eixo da semente (16,57mm e 17,88mm), evidenciando que há diferenciação nos parâmetros avaliados de uma 
safra para outra em populações nativas. Tonini et al. (2014) ao avaliarem variações anuais na produção de frutos e sementes de castanheira-do-brasil em florestas nativas de Roraima constataram que há variação na produção anual nas populações e entre os indivíduos na mesma população.

Houve variação de 0,11 a 0,84, com média de 0,32, nas distâncias entre os pares de matrizes. Foi encontrada a maior divergência entre as matrizes ITA5 e ITA19, com 0,84. A menor dissimilaridade registrada neste estudo foi de 0,11 entre ITA1 e ITA8. Todas as combinações entre as matrizes listadas na tabela 3, podem promover grande heterogeneidade, além de maior diversidade genética nas gerações futuras.

Tabela 3: Pares de matrizes mais divergentes estimados a partir da Distância Euclidiana Média no estudo sobre divergência entre as 21 matrizes de Castanheira-do-Brasil.

\begin{tabular}{|c|c|c|}
\hline Ordem & Distância Euclidiana Média & Pares de Genótipos \\
\hline 10 & 0,84 & ITA19 x ITA05 \\
\hline 20 & 0,74 & ITA02 x ITA05 \\
\hline 3응 & 0,71 & ITA10 x ITA05 \\
\hline 40 & 0,70 & ITA12 x ITA05 \\
\hline 50 & 0,69 & ITA20 x ITA05 \\
\hline 60 & 0,66 & ITA13 x ITA05 \\
\hline 70 & 0,65 & ITA18 x ITA05 \\
\hline 80 & 0,65 & ITA09 x ITA05 \\
\hline 9응 & 0,64 & ITA01 x ITA05 \\
\hline
\end{tabular}

A matriz ITA5 esteve presente em todas as combinações mais divergentes (tabela 3). A matriz ITA19, que juntamente com ITA5 forma o par mais divergente possui a maior média de massa total do fruto (MTF) e maior média do diâmetro longitudinal do fruto (DLF). A matriz ITA2 possui as maiores médias para características das sementes, como massa unitária da semente (MUS), maior eixo da semente (MAS) e eixo médio da semente (EMS).

A matriz ITA10 apresentou uma maior média para a massa fresca das sementes (MFS). Observando os nove pares de matrizes que mais divergiram, as três primeiras combinações são as melhores em relação aos desempenhos dos caracteres, ITA19 x ITA5; ITA02 x ITA5; ITA10 x ITA5 (tabela 3). Na Tabela 4 está representada a distância euclidiana média entre as 21 matrizes de Castanheira-do-Brasil, calculada a partir dos 12 caracteres avaliados.

Tabela 4: Distância euclidiana média entre 21 matrizes de castanheira-do-brasil de populações naturais, calculada a partir de 12 caracteres.

\begin{tabular}{|c|c|c|c|c|c|c|c|c|c|c|c|c|c|c|c|c|c|c|c|c|c|}
\hline & $\begin{array}{l}\text { IT } \\
\text { A } \\
1\end{array}$ & $\begin{array}{l}\text { IT } \\
\text { A } \\
2\end{array}$ & $\begin{array}{l}\text { IT } \\
\text { A } \\
3\end{array}$ & $\begin{array}{l}\text { IT } \\
\text { A } \\
4\end{array}$ & $\begin{array}{l}\text { IT } \\
\text { A } \\
5\end{array}$ & $\begin{array}{l}\text { IT } \\
\text { A } \\
6\end{array}$ & $\begin{array}{l}\text { IT } \\
\text { A } \\
7\end{array}$ & $\begin{array}{l}\text { IT } \\
\text { A } \\
8\end{array}$ & $\begin{array}{l}\text { IT } \\
\text { A } \\
9\end{array}$ & $\begin{array}{l}\text { IT } \\
\text { A1 } \\
0\end{array}$ & $\begin{array}{l}\text { IT } \\
\text { A1 } \\
1\end{array}$ & $\begin{array}{l}\text { IT } \\
\text { A1 } \\
2\end{array}$ & $\begin{array}{l}\text { IT } \\
\text { A1 } \\
3\end{array}$ & $\begin{array}{l}\text { IT } \\
\text { A1 } \\
4\end{array}$ & $\begin{array}{l}\text { IT } \\
\text { A1 } \\
5\end{array}$ & $\begin{array}{l}\text { IT } \\
\text { A1 } \\
6\end{array}$ & $\begin{array}{l}\text { IT } \\
\text { A1 } \\
7\end{array}$ & $\begin{array}{l}\text { IT } \\
\text { A1 } \\
8\end{array}$ & $\begin{array}{l}\text { IT } \\
\text { A1 } \\
9\end{array}$ & $\begin{array}{l}\text { IT } \\
\text { A2 } \\
0\end{array}$ & $\begin{array}{l}\text { IT } \\
\text { A2 } \\
1\end{array}$ \\
\hline $\begin{array}{l}\text { IT } \\
\text { A1 }\end{array}$ & & & & & & & & & & & & & & & & & & & & & \\
\hline $\begin{array}{l}\text { IT } \\
\text { A2 }\end{array}$ & $\begin{array}{l}0, \\
16\end{array}$ & & & & & & & & & & & & & & & & & & & & \\
\hline $\begin{array}{l}\text { IT } \\
\text { A3 }\end{array}$ & $\begin{array}{l}0, \\
42\end{array}$ & $\begin{array}{l}0, \\
46\end{array}$ & & & & & & & & & & & & & & & & & & & \\
\hline $\begin{array}{l}\text { IT } \\
\text { A4 }\end{array}$ & $\begin{array}{l}0, \\
42\end{array}$ & $\begin{array}{l}0, \\
46\end{array}$ & $\begin{array}{l}0, \\
27\end{array}$ & & & & & & & & & & & & & & & & & & \\
\hline $\begin{array}{l}\text { IT } \\
\text { A5 }\end{array}$ & $\begin{array}{l}0, \\
64\end{array}$ & $\begin{array}{l}0, \\
74\end{array}$ & $\begin{array}{l}0, \\
44 \\
\end{array}$ & $\begin{array}{l}0, \\
42 \\
\end{array}$ & & & & & & & & & & & & & & & & & \\
\hline $\begin{array}{l}\text { IT } \\
\text { A6 }\end{array}$ & $\begin{array}{l}0, \\
34\end{array}$ & $\begin{array}{l}0, \\
41\end{array}$ & $\begin{array}{l}0, \\
28\end{array}$ & $\begin{array}{l}0, \\
39\end{array}$ & $\begin{array}{l}0, \\
57\end{array}$ & & & & & & & & & & & & & & & & \\
\hline $\begin{array}{l}\text { IT } \\
\text { A7 }\end{array}$ & $\begin{array}{l}0, \\
18\end{array}$ & $\begin{array}{l}0, \\
31\end{array}$ & $\begin{array}{l}0 \\
28\end{array}$ & $\begin{array}{l}0, \\
35\end{array}$ & $\begin{array}{l}0, \\
52\end{array}$ & $\begin{array}{l}0, \\
21\end{array}$ & & & & & & & & & & & & & & & \\
\hline
\end{tabular}




\begin{tabular}{|c|c|c|c|c|c|c|c|c|c|c|c|c|c|c|c|c|c|c|c|c|}
\hline $\begin{array}{l}\text { IT } \\
\text { A8 }\end{array}$ & $\begin{array}{l}0, \\
11\end{array}$ & $\begin{array}{l}0, \\
23\end{array}$ & $\begin{array}{l}0, \\
35\end{array}$ & $\begin{array}{l}0, \\
37\end{array}$ & $\begin{array}{l}0, \\
60\end{array}$ & $\begin{array}{l}0, \\
26\end{array}$ & $\begin{array}{l}0, \\
12\end{array}$ & & & & & & & & & & & & & \\
\hline $\begin{array}{l}\text { IT } \\
\text { A9 }\end{array}$ & $\begin{array}{l}0, \\
26 \\
\end{array}$ & $\begin{array}{l}0, \\
23 \\
\end{array}$ & $\begin{array}{l}0, \\
30 \\
\end{array}$ & $\begin{array}{l}0 \\
38 \\
\end{array}$ & $\begin{array}{l}0, \\
65 \\
\end{array}$ & $\begin{array}{l}0, \\
31 \\
\end{array}$ & $\begin{array}{l}, \\
26 \\
\end{array}$ & $\begin{array}{l}0, \\
27\end{array}$ & & & & & & & & & & & & \\
\hline $\begin{array}{l}\text { IT } \\
\text { A1 } \\
0\end{array}$ & $\begin{array}{l}0, \\
22\end{array}$ & $\begin{array}{l}0, \\
25\end{array}$ & $\begin{array}{l}0, \\
46\end{array}$ & $\begin{array}{l}0, \\
52\end{array}$ & $\begin{array}{l}0, \\
71\end{array}$ & $\begin{array}{l}0, \\
36\end{array}$ & $\begin{array}{l}0, \\
27\end{array}$ & $\begin{array}{l}0, \\
22\end{array}$ & $\begin{array}{l}0, \\
34\end{array}$ & & & & & & & & & & & \\
\hline $\begin{array}{l}\text { IT } \\
\text { A1 } \\
1\end{array}$ & $\begin{array}{l}0, \\
49\end{array}$ & $\begin{array}{l}0, \\
58\end{array}$ & $\begin{array}{l}0, \\
30\end{array}$ & $\begin{array}{l}0, \\
39\end{array}$ & $\begin{array}{l}0, \\
32\end{array}$ & $\begin{array}{l}0, \\
35\end{array}$ & $\begin{array}{l}0, \\
34\end{array}$ & $\begin{array}{l}0, \\
42\end{array}$ & $\begin{array}{l}0, \\
51\end{array}$ & $\begin{array}{l}0,4 \\
8\end{array}$ & & & & & & & & & & \\
\hline $\begin{array}{l}\text { IT } \\
\text { A1 } \\
2 \\
\end{array}$ & $\begin{array}{l}0, \\
22\end{array}$ & $\begin{array}{l}0, \\
21\end{array}$ & $\begin{array}{l}0, \\
37\end{array}$ & $\begin{array}{l}0 \\
45\end{array}$ & $\begin{array}{l}0 \\
70\end{array}$ & $\begin{array}{l}0 \\
25\end{array}$ & $\begin{array}{l}0, \\
22\end{array}$ & $\begin{array}{l}0, \\
21\end{array}$ & $\begin{array}{l}0, \\
18\end{array}$ & $\begin{array}{l}0,2 \\
3\end{array}$ & $\begin{array}{l}0,5 \\
0\end{array}$ & & & & & & & & & \\
\hline $\begin{array}{l}\text { IT } \\
\text { A1 } \\
\mathbf{3}\end{array}$ & $\begin{array}{l}0, \\
23\end{array}$ & $\begin{array}{l}0, \\
21\end{array}$ & $\begin{array}{l}0, \\
35\end{array}$ & $\begin{array}{l}0, \\
42\end{array}$ & $\begin{array}{l}0, \\
66\end{array}$ & $\begin{array}{l}0 \\
29\end{array}$ & $\begin{array}{l}0 \\
24\end{array}$ & $\begin{array}{l}0 \\
22\end{array}$ & $\begin{array}{l}0 \\
19\end{array}$ & $\begin{array}{l}0,1 \\
8\end{array}$ & $\begin{array}{l}0,4 \\
5\end{array}$ & $\begin{array}{l}0,1 \\
1\end{array}$ & & & & & & & & \\
\hline $\begin{array}{l}\text { IT } \\
\text { A1 } \\
4 \\
\end{array}$ & $\begin{array}{l}0, \\
32\end{array}$ & $\begin{array}{l}0, \\
41\end{array}$ & $\begin{array}{l}0, \\
16\end{array}$ & $\begin{array}{l}0, \\
26\end{array}$ & $\begin{array}{l}0, \\
38\end{array}$ & $\begin{array}{l}0, \\
26\end{array}$ & $\begin{array}{l}0, \\
18\end{array}$ & $\begin{array}{l}0, \\
27\end{array}$ & $\begin{array}{l}0, \\
30\end{array}$ & $\begin{array}{l}0,4 \\
0\end{array}$ & $\begin{array}{l}0,2 \\
3\end{array}$ & $\begin{array}{l}0,3 \\
4\end{array}$ & $\begin{array}{l}0,3 \\
2\end{array}$ & & & & & & & \\
\hline $\begin{array}{l}\text { IT } \\
\text { A1 } \\
5\end{array}$ & $\begin{array}{l}0, \\
22\end{array}$ & $\begin{array}{l}0, \\
32\end{array}$ & $\begin{array}{l}0, \\
24\end{array}$ & $\begin{array}{l}0, \\
28\end{array}$ & $\begin{array}{l}0, \\
47\end{array}$ & $\begin{array}{l}0, \\
26\end{array}$ & $\begin{array}{l}0, \\
12\end{array}$ & $\begin{array}{l}0, \\
16\end{array}$ & $\begin{array}{l}0, \\
28\end{array}$ & $\begin{array}{l}0,2 \\
8\end{array}$ & $\begin{array}{l}0,2 \\
8\end{array}$ & $\begin{array}{l}0,2 \\
8\end{array}$ & $\begin{array}{l}0,2 \\
5\end{array}$ & $\begin{array}{l}0,1 \\
5\end{array}$ & & & & & & \\
\hline $\begin{array}{l}\text { IT } \\
\text { A1 } \\
6\end{array}$ & $\begin{array}{l}0, \\
32\end{array}$ & $\begin{array}{l}0, \\
36\end{array}$ & $\begin{array}{l}0, \\
14\end{array}$ & $\begin{array}{l}0 \\
28\end{array}$ & $\begin{array}{l}0, \\
50\end{array}$ & $\begin{array}{l}0 \\
20\end{array}$ & $\begin{array}{l}0 \\
19\end{array}$ & $\begin{array}{l}0 \\
25\end{array}$ & $\begin{array}{l}0 \\
23\end{array}$ & $\begin{array}{l}0,3 \\
3\end{array}$ & $\begin{array}{l}0,3 \\
0\end{array}$ & $\begin{array}{l}0,2 \\
5\end{array}$ & $\begin{array}{l}0,2 \\
2\end{array}$ & $\begin{array}{l}0,1 \\
6\end{array}$ & $\begin{array}{l}0,1 \\
6\end{array}$ & & & & & \\
\hline $\begin{array}{l}\text { IT } \\
\text { A1 } \\
7\end{array}$ & $\begin{array}{l}0, \\
29\end{array}$ & $\begin{array}{l}0, \\
29\end{array}$ & $\begin{array}{l}0, \\
29\end{array}$ & $\begin{array}{l}0, \\
32\end{array}$ & $\begin{array}{l}0, \\
53\end{array}$ & $\begin{array}{l}0, \\
39\end{array}$ & $\begin{array}{l}0, \\
28\end{array}$ & $\begin{array}{l}0, \\
30\end{array}$ & $\begin{array}{l}0, \\
20\end{array}$ & $\begin{array}{l}0,3 \\
7\end{array}$ & $\begin{array}{l}0,4 \\
2\end{array}$ & $\begin{array}{l}0,3 \\
0\end{array}$ & $\begin{array}{l}0,2 \\
5\end{array}$ & $\begin{array}{l}0,2 \\
3\end{array}$ & $\begin{array}{l}0,2 \\
4\end{array}$ & $\begin{array}{l}0,2 \\
5\end{array}$ & & & & \\
\hline $\begin{array}{l}\text { IT } \\
\text { A1 } \\
8\end{array}$ & $\begin{array}{l}0, \\
18\end{array}$ & $\begin{array}{l}0, \\
20\end{array}$ & $\begin{array}{l}0, \\
33\end{array}$ & $\begin{array}{l}0, \\
41\end{array}$ & $\begin{array}{l}0, \\
65\end{array}$ & $\begin{array}{l}0, \\
25\end{array}$ & $\begin{array}{l}0, \\
18\end{array}$ & $\begin{array}{l}0, \\
15\end{array}$ & $\begin{array}{l}0, \\
18\end{array}$ & $\begin{array}{l}0,2 \\
0\end{array}$ & $\begin{array}{l}0,4 \\
6\end{array}$ & $\begin{array}{l}0,1 \\
1\end{array}$ & $\begin{array}{l}0,1 \\
3\end{array}$ & $\begin{array}{l}0,3 \\
0\end{array}$ & $\begin{array}{l}0,2 \\
2\end{array}$ & $\begin{array}{l}0,2 \\
1\end{array}$ & $\begin{array}{l}0,2 \\
9\end{array}$ & & & \\
\hline $\begin{array}{l}\text { IT } \\
\text { A1 } \\
9\end{array}$ & $\begin{array}{l}0, \\
32\end{array}$ & $\begin{array}{l}0, \\
32\end{array}$ & $\begin{array}{l}0, \\
56\end{array}$ & $\begin{array}{l}0, \\
62\end{array}$ & $\begin{array}{l}0, \\
84\end{array}$ & $\begin{array}{l}0, \\
41\end{array}$ & $\begin{array}{l}0, \\
37\end{array}$ & $\begin{array}{l}0, \\
32\end{array}$ & $\begin{array}{l}0, \\
40\end{array}$ & $\begin{array}{l}0,1 \\
7\end{array}$ & $\begin{array}{l}0,6 \\
0\end{array}$ & $\begin{array}{l}0,2 \\
6\end{array}$ & $\begin{array}{l}0,2 \\
7\end{array}$ & $\begin{array}{l}0,5 \\
2\end{array}$ & $\begin{array}{l}0,4 \\
1\end{array}$ & $\begin{array}{l}0,4 \\
2\end{array}$ & $\begin{array}{l}0,5 \\
0\end{array}$ & $\begin{array}{l}0,2 \\
5\end{array}$ & & \\
\hline $\begin{array}{l}\text { IT } \\
\text { A2 } \\
0\end{array}$ & $\begin{array}{l}0, \\
26\end{array}$ & $\begin{array}{l}0, \\
35\end{array}$ & $\begin{array}{l}0, \\
47\end{array}$ & $\begin{array}{l}0, \\
53\end{array}$ & $\begin{array}{l}0, \\
69\end{array}$ & $\begin{array}{l}0, \\
34\end{array}$ & $\begin{array}{l}0, \\
26\end{array}$ & $\begin{array}{l}0, \\
22\end{array}$ & $\begin{array}{l}0, \\
40\end{array}$ & $\begin{array}{l}0,1 \\
5\end{array}$ & $\begin{array}{l}0,4 \\
6\end{array}$ & $\begin{array}{l}0,2 \\
9\end{array}$ & $\begin{array}{l}0,2 \\
7\end{array}$ & $\begin{array}{l}0,4 \\
0\end{array}$ & $\begin{array}{l}0,2 \\
8\end{array}$ & $\begin{array}{l}0,3 \\
4\end{array}$ & $\begin{array}{l}0,4 \\
4\end{array}$ & $\begin{array}{l}0,2 \\
3\end{array}$ & $\begin{array}{l}0.1 \\
9\end{array}$ & \\
\hline $\begin{array}{l}\text { IT } \\
\text { A2 } \\
1\end{array}$ & $\begin{array}{l}0, \\
21\end{array}$ & $\begin{array}{l}0, \\
27\end{array}$ & $\begin{array}{l}0 \\
37\end{array}$ & $\begin{array}{l}0 \\
42\end{array}$ & $\begin{array}{l}0, \\
61\end{array}$ & $\begin{array}{l}0 \\
28\end{array}$ & $\begin{array}{l}0 \\
20\end{array}$ & $\begin{array}{l}0, \\
16\end{array}$ & $\begin{array}{l}0, \\
31\end{array}$ & $\begin{array}{l}0,1 \\
2\end{array}$ & $\begin{array}{l}0,3 \\
7\end{array}$ & $\begin{array}{l}0,2 \\
2\end{array}$ & $\begin{array}{l}0,1 \\
7\end{array}$ & $\begin{array}{l}0,2 \\
9\end{array}$ & $\begin{array}{l}0,1 \\
8\end{array}$ & $\begin{array}{l}0,2 \\
4\end{array}$ & $\begin{array}{l}0,3 \\
2\end{array}$ & $\begin{array}{l}0,1 \\
8\end{array}$ & $\begin{array}{l}0,2 \\
6\end{array}$ & $\begin{array}{l}0,1 \\
7\end{array}$ \\
\hline
\end{tabular}

A análise de agrupamento hierárquico pelo método UPGMA, utilizando 12 características quantitativas, baseado na distância euclidiana média proporcionou a formação de três grupos (figura 1). A matriz ITA19 está alocada no grupo I, porém observa-se que ele se encontra parcialmente separado formando um subgrupo exclusivo neste grupo.

O grupo I apresentou maior número de matrizes quando comparado ao grupo II, onde foram alocados seis matrizes (ITA3; ITA16; ITA14; ITA6; ITA17 e ITA4) e ao grupo III, que alocou ITA5 e ITA11, sendo estas as mais distantes das outras pois se agruparam com as demais em $100 \%$ de dissimilaridade. As dissimilaridades genéticas entre as 21 matrizes de castanheira-do-brasil variaram de 0,844 a 0,113. Verificase que a menor similaridade ocorreu entre ITA1 e ITA8 $(0,113)$, enquanto a maior dissimilaridade foi entre ITA5 e ITA19 $(0,844)$.

No agrupamento pelo método de otimização de Tocher verificou-se a formação de quatro grupos (tabela 5), sendo que o grupo I possui a maior quantidade de matrizes, correspondendo a $76,19 \%$. Os grupos II e III agruparam duas matrizes cada, sendo eles ITA3 e ITA4, as quais apresentaram baixas médias para o caractere massa seca das sementes e ITA5 e ITA11, que apresentaram baixas médias para a maioria dos 
caracteres das sementes. O grupo IV foi constituído por apenas uma matriz (ITA19), que apresentou elevadas médias para as características do fruto, principalmente a massa do fruto.

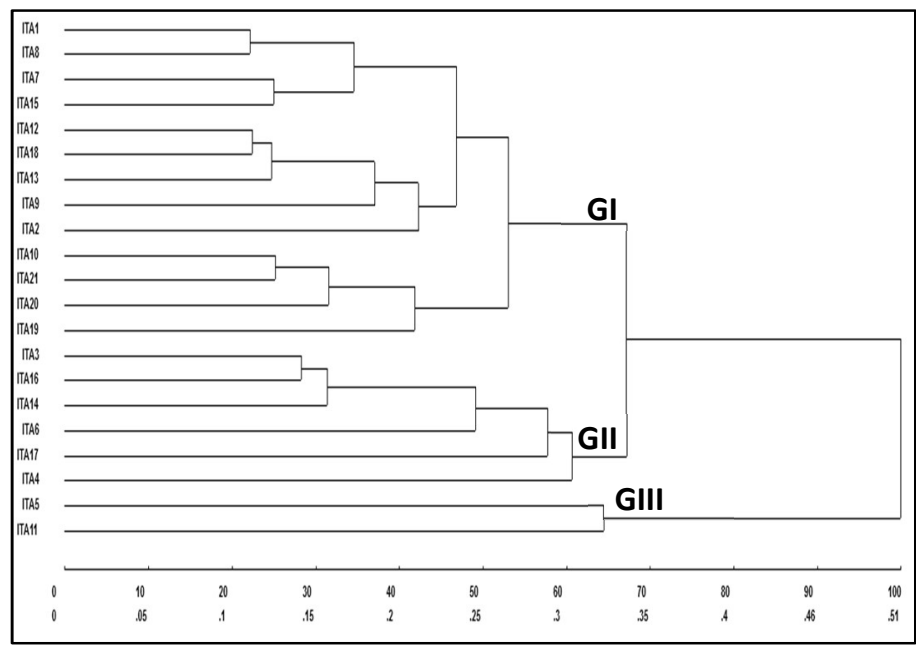

Figura 1: Dendrograma resultante da análise de 21 matrizes de castanheira-do-brasil, obtido pelo método de agrupamento UPGMA, utilizando a distância euclidiana média como medida de distância genética. Coeficiente de correlação cofenética $(0,7511)$.

Tabela 5: Agrupamento das 21 matrizes de Castanheira-do-Brasil, pelo método de agrupamento de Tocher, utilizando a distância Euclidiana média como medida de distância genética.

\begin{tabular}{|l|l|}
\hline \multicolumn{1}{|c|}{ Grupos } & \multicolumn{1}{|c|}{ Genótipos } \\
\hline I & ITA1 ITA8 ITA7 ITA15 ITA18 ITA21 ITA13 ITA12 ITA10 ITA20 ITA2 ITA16 ITA9 ITA6 ITA14 ITA17 \\
\hline II & ITA3 ITA4 \\
\hline III & ITA5 ITA11 \\
\hline IV & ITA19 \\
\hline
\end{tabular}

Este tipo de agrupamento se baseia na formação de grupos cujas distâncias intergrupos sejam menores que as distâncias intragrupos. Ao final do processo, obtém-se o número de grupos e os acessos contidos em cada grupo, geralmente acarretando uma maior concentração de genótipos nos primeiros grupos (FARIA et al., 2012).

Ao comparar os agrupamentos obtidos pelos métodos de UPGMA e Tocher percebe-se resultados similares, sendo o primeiro grupo constituído pela maioria das matrizes. As matrizes ITA5 e ITA11 foram agrupadas em um único grupo em ambos os métodos de agrupamento e ITA4 também ficou distanciada do grupo principal.

A análise dos componentes principais revelou que os três primeiros componentes absorveram $86,41 \%$ do total da variação, sendo que o primeiro componente explicou $59,68 \%$, o segundo componente $17,35 \%$ e o terceiro $9,36 \%$ da variação total (tabela 6). Geralmente, descritores com maiores autovetores e maiores coeficientes de correlação são considerados de maior importância para o respectivo componente (IEZZONI et al., 1991). Os resultados foram diferentes dos encontrados por Teixeira et al. (2015) ao avaliar genótipos de Castanheira-do-Brasil, onde os três primeiros componentes principais explicaram cerca de 77\% da variação investigada.

Tabela 6: Estimativas dos autovalores associados aos componentes principais, juntamente com sua importância relativa (\%) e acumulada (\%), referentes a 12 características morfológicas de Castanheira-do-Brasil.

\begin{tabular}{|c|c|c|c|}
\hline $\mathbf{C P}^{1}$ & Autovalor & Importância Relativa (\%) & (\%) Acumulada \\
\hline $\mathbf{C P}_{\mathbf{1}}$ & 7,16 & 59,68 & $\mathbf{5 9 , 6 8}$ \\
\hline
\end{tabular}




\begin{tabular}{|l|l|c|c|}
\hline $\mathbf{C P}_{\mathbf{2}}$ & 2,08 & 17,35 & $\mathbf{7 7 , 0 4}$ \\
\hline $\mathbf{C P}_{\mathbf{3}}$ & 1,12 & 9,36 & $\mathbf{8 6 , 4 1}$ \\
\hline $\mathbf{C P}_{\mathbf{4}}$ & 0,63 & 5,28 & 91,69 \\
\hline $\mathbf{C P}_{\mathbf{5}}$ & 0,44 & 3,74 & 95,43 \\
\hline $\mathbf{C P}_{\mathbf{6}}$ & 0,24 & 2,02 & 97,46 \\
\hline $\mathbf{C P}_{\mathbf{7}}$ & 0,17 & 1,47 & 98,94 \\
\hline $\mathbf{C P}$ & 0,07 & 0,66 & 99,60 \\
\hline $\mathbf{C P}$ & 0,02 & 0,21 & 99,82 \\
\hline $\mathbf{C P}_{10}$ & 0,01 & 0,10 & 99,92 \\
\hline $\mathbf{C P}_{11}$ & 0,00 & 0,05 & 99,97 \\
\hline $\mathbf{C P}_{12}$ & 0,00 & 0,02 & 100,00 \\
\hline
\end{tabular}

Legenda: ${ }^{1}$ Cada componente principal é uma combinação linear das doze variáveis padronizadas.

O componente principal $C P^{1}$, que explicou $59,68 \%$ da variância total, está associado a um contraste entre grupos de variáveis, com maior peso neste componente os caracteres: massa fresca das sementes, massa seca das sementes, massa unitária da semente e maior eixo da semente, contribuindo para a variabilidade das variáveis com $0,32 \%$ para todas as variáveis citadas (tabela 7), sendo variáveis mais significativas a seleção de matrizes para a produção de sementes.

Tabela 7: Conjunto dos autovetores (coeficiente de ponderação) explicadas pelos componentes principais (CPi) das 12 variáveis analisadas em Castanheira-do-Brasil.

\begin{tabular}{|c|c|c|c|c|c|c|c|c|c|c|c|c|}
\hline \multirow[b]{2}{*}{$C p_{1}$} & \multicolumn{12}{|c|}{ Elementos dos autovetores associados } \\
\hline & MTF & DLF & DTF & ECF & NSF & MFS & MSS & MUS & MAS & EMS & MES & MUA \\
\hline $\mathrm{CP}_{1}$ & 0,28 & 0,29 & 0,31 & 0,23 & 0,10 & 0,32 & 0,32 & 0,32 & 0,32 & 0,30 & 0,25 & 0,29 \\
\hline $\mathrm{CP}_{\mathbf{2}}$ & 0,31 & 0,27 & 0,26 & 0,10 & 0,54 & 0,10 & 0,10 & $-0,31$ & $-0,24$ & $-0,32$ & $-0,39$ & $-0,07$ \\
\hline $\mathrm{CP}_{3}$ & $-0,30$ & $-0,36$ & $-0,31$ & $-0,28$ & 0,46 & 0,42 & 0,42 & 0,01 & 0,08 & 0,00 & 0,00 & $-0,01$ \\
\hline $\mathrm{CP}_{4}$ & $-0,21$ & $-0,16$ & $-0,08$ & 0,83 & 0,10 & 0,03 & 0,01 & $-0,12$ & 0,07 & 0,13 & 0,05 & $-0,40$ \\
\hline $\mathrm{CP}_{5}$ & $-0,11$ & $-0,20$ & $-0,15$ & 0,35 & $-0,11$ & $-0,06$ & 0,08 & 0,11 & 0,04 & $-0,30$ & $-0,39$ & 0,70 \\
\hline $\mathrm{CP}_{6}$ & 0,14 & $-0,14$ & $-0,05$ & 0,13 & 0,03 & 0,00 & 0,12 & $-0,04$ & $-0,67$ & $-0,17$ & 0,62 & 0,21 \\
\hline $\mathrm{CP}_{7}$ & $-0,50$ & 0,30 & 0,08 & $-0,01$ & 0,50 & $-0,36$ & $-0,26$ & $-0,04$ & 0,16 & $-0,03$ & 0,29 & 0,26 \\
\hline $\mathrm{CP}_{8}$ & $-0,03$ & $-0,03$ & $-0,03$ & $-0,01$ & 0,08 & 0,00 & $-0,10$ & $-0,28$ & $-0,34$ & 0,79 & $-0,25$ & 0,28 \\
\hline $\mathrm{CP}_{9}$ & 0,49 & 0,10 & $-0,69$ & 0,05 & 0,22 & 0,06 & $-0,38$ & $-0,02$ & 0,20 & 0,00 & 0,09 & 0,07 \\
\hline $\mathrm{CP}_{10}$ & $-0,15$ & 0,57 & $-0,44$ & 0,03 & $-0,08$ & $-0,24$ & 0,45 & 0,26 & $-0,24$ & 0,06 & $-0,14$ & $-0,14$ \\
\hline $\mathrm{CP}_{11}$ & 0,25 & $-0,39$ & 0,10 & $-0,01$ & 0,36 & $-0,48$ & 0,00 & 0,58 & $-0,08$ & 0,13 & $-0,13$ & $-0,13$ \\
\hline $\mathrm{CP}_{12}$ & 0,24 & $-0,14$ & $-0,04$ & $-0,05$ & $-0,03$ & $-0,50$ & 0,48 & $-0,52$ & 0,33 & 0,05 & 0,14 & 0,06 \\
\hline
\end{tabular}

Legenda: MTF: massa total do fruto (g), DLF: diâmetro longitudinal do fruto (mm), DTF: diâmetro transversal do fruto $(\mathrm{mm})$, ECF: espessura da casca do fruto $(\mathrm{mm}), \mathrm{NSF}$ : número de sementes por fruto, MFS: massa fresca das sementes (g), MSS: massa seca das sementes (g), MUS: massa unitária da semente (g), MAS: maior eixo da semente (mm), EMS: eixo médio da semente $(\mathrm{mm})$, MES: menor eixo da semente $(\mathrm{mm})$ e MUA: massa unitária da amêndoa (g).

Para o componente $\mathrm{CP}^{2}$, que explicou $17,35 \%$ da variância total, destacaram-se o número de sementes por fruto, o eixo médio da semente e o menor eixo da semente. E no terceiro, novamente aparecem os caracteres número de sementes por fruto, massa fresca das sementes e massa seca das sementes (tabela 7). A partir da análise dos caracteres nos três primeiros componentes principais pode-se observar que as características avaliadas nas sementes foram as que mais contribuíram, comprovando a importância de avaliá-las em trabalhos relacionados a diversidade genética de matrizes de Castanheira-doBrasil.

\section{CONCLUSÕES}

Os caracteres de maior contribuição para a variabilidade genética dos genótipos estudados são: número de sementes por fruto (NSF), massa fresca das sementes (MFS), massa seca das sementes (MSS), 
menor eixo das sementes (MES) e massa unitária das amêndoas (MUA). Portanto, estes caracteres são considerados os mais responsivos para a seleção de genótipos da espécie em estudo.

Há divergência genética entre as árvores matrizes de castanheira-do-brasil analisadas. As matrizes que apresentaram características mais desejáveis podem ser utilizadas para a propagação de mudas ou seleção de gemas para bancos de germoplasma e futuramente para produtores que tenham o intuito de reflorestar áreas degradas ou realizar plantios comerciais de Castanheira-do-Brasil.

\section{REFERÊNCIAS}

BRAGA, E. T. M.. Diversidade morfológica e produção de Bertholletia excelsa HBK (Lecythidaceae) no sudeste do estado do Acre-Brasil. Dissertação (Mestrado em Ecologia e Manejo de Recursos Naturais) - Universidade Federal do Acre, Rio Branco, 2007.

CAMARGO, F. F.; COSTA, R. B.; RESENDE, M. D. V.; ROA, R. A. R.; RODRIGUES, N. B.; SANTOS, L. V.; FREITAS, A. C. A.. Variabilidade genética para caracteres morfométricos de matrizes de castanha-do-brasil da Amazônia Matogrossense. Acta Amazônica, Manaus, v.40, n.4, p.705-710, 2010. DOI: http://doi.org/10.1590/S0044$\underline{59672010000400010}$

CARVALHO, J. E. U.; NAZARÉ, R. F. R.; OLIVEIRA, W. M. Características físicas e físico-químicas de um tipo de bacuri (Platonia insignis Mart.) com rendimento industrial superior. Revista Brasileira de Fruticultura, Jaboticabal, v.25, p.326328, 2003. DOI: http://doi.org/10.1590/S0100$\underline{29452003000200036}$

CLAY, J. W.. Brazil nuts: The use of a keystone species for conservation and development. In: FREESE, C. H.. Harvesting wild species. Implications for biodiversity conservation. Baltimore: The John Hopking University Press, 1997. p.246282.

CLEMENT, C. R.. Castanha-do-Pará (Bertholletia excelsa). In: CLAY, W. J.; SAMPAIO, T. B.; CLEMENT, C. R.. Biodiversidade amazônica: exemplos e estratégias de utilização. Manaus: SEBRAEPDET, 1999. p.118-131.

CRUZ, C. D.. Programa Genes: Biometria. Viçosa: EDUFV, 2006.

CRUZ, E. D.; CARVALHO, J. E. U.. Biometria de frutos e sementes e germinação de curupixá (Micropholis cf. venulosa Mart. \& Eichler - Sapotaceae). Acta Amazônica, Manaus, v.33, n.3, p.389-398, 2003.

CRUZ, E. D.; MARTINS, F. O.; CARVALHO, J. E. U. Biometria de frutos e sementes de jatobá-curuba (Hymenaea intermedia Ducke, Leguminosae Caesalpinioideae). Revista Brasileira de Botânica, São Paulo, v.24, p.161-165, 2001. DOI: http://doi.org/10.1590/S0100-84042001000200005

EDWARDS, D. G. W.. Forest tree seeds at the end of 20th century: major accomplishments and needs. In: FORESTS AND SOCIETY: THE ROLE OF RESEARCH: IUFRO WORLD CONGRESS, 21. Anais. Kuala Lumpur: 2000.

FARIA, P. N.; CECON, P. R.; SILVA, A. R.; FINGER, F. L.; SILVA, F.; CRUZ, C. D.; SÁVIO, F. L.. Métodos de agrupamento em estudo de divergência genética de pimentas. Horticultura Brasileira, Vitória da Conquista, v.30, n.3, p.428-432, 2012.

IEZZONI, A. F.; PRITTS, M. P.. Applications of principal component analysis to horticultural research. HortScience, Alexandria, v.26, n.4, p.334-338, 1991.

KAMINSKI, P. E.; TONINI, H.; JÚNIOR, M. M.; SCHWENGBER, L. A. M.. Biometria de Frutos de Castanha-do-brasil (Bertholletia excelsa Bonpl.). Manejo Sustentável do Produtos Florestais Não-madeireiros na Amazônia, Roraima, v.1, p.45-52, 2008.

MELO, M. G. G.; MENDONÇA, M. S.; MENDES, A. M. S.. Análise morfológica de sementes, germinação e plântulas de jatobá (Hymenaea intermedia Ducke var. adenotricha (Ducke) Lee \& Lang.) (Leguminosae-caesalpinioideae). Acta Amazônica, Manaus, v.34, n.1, p.9-14, 2004. DOI: http://doi.org/10.1590/S0044-59672004000100002

MORI, S. A.; PRANCE, G. T.. Taxonomy, ecology, and economic botany of the Brazil nut (Bertholletia excelsa Humb. \& Bonpl.: Lecythidaceae). Advances in Economic Botany, New York, v.8, p.130-150, 1990.

NOGUEIRA, R. M.; ÁLVARES, V. D. S.; RUFFATO, S.; LOPES, R. P.; SILVA, J. D. S.. Physical properties of Brazil

nuts. Engenharia Agrícola, Jaboticabal, v.34, n.5, p.963-971, 2014. DOI: http://doi.org/10.1590/S0100$\underline{69162014000500015}$

O'MALLEY D. M.; BUCKLEY, D. P.; PRANCE, G. T.; BAWA, K. S.. Genetic of Brasil nut (Bertholletia excelsa Humb. \& Bonpl., Lecythidaceae). Theoretical and Applied Genetics, Berlin, v.76, n.1, p.929-932, 1988.

ORTIZ, E. G.. Brazil nut (Bertholletia excelsa). Tapping the green market: certification and management of non-timber forest products. London: 2002.

PAULA, R. C.. Repetibilidade e divergência genética entre matrizes de Pterogyne nitens Tul. (Fabaceae -

Caesalpinioideae) por caracteres biométricos de frutos e de sementes e parâmetros da qualidade fisiológica de sementes. Tese (Doutorado em Silvicultura) - Universidade Estadual Paulista Julio de Mesquita Filho, Jaboticabal, 2007.

PIÑA-RODRIGUES, F. C. M.; AGUIAR, I. B.. Maturação e dispersão de sementes. In: AGUIAR, I. B; PIÑA-RODRIGUES, F. C. M.; FIGLIOLIA, M. B.. Sementes florestais tropicais. Brasília: ABRATES, 1993. p.215-274. 
RAO, C. R. Advanced statistical methods in biometric research. New York: 1952.

SNEATH, P. H.; SOKAL, R. R.. Numerical taxonomy: The principles and practice of numerical classification. San Francisco: 1973.

SOKAL, R. R.; ROHLF, F. J.. The comparison of dendrograms by objective methods. Taxon, Utrecht, v.11, n.2, p.33-40, 1962. DOI: http://doi.org/10.2307/1217208

TAFFAREL, C.; BOTELHO, S. C. C.; BOTIN, A. A.; BOTELHO, F. M.; TARDIN, A. B. B.. Fatores de forma de sementes de castanheira-do-brasil do estado de Mato Grosso. In: CONGRESSO BRASILEIRO DE ENGENHARIA AGRÍCOLA, 42. Anais. Fortaleza: 2013.

TEIXEIRA, R. A.; PEDROZO, C. Â.; COSTA, E. K. L.; BATISTA, K. D.; TONINI, H.; PESSONI, L. A.. Correlation and phenotypic divergence among cultivated genotypes of Brazil nut. Scientia Forestalis, Piracicaba, v.43, n.107, p.523-531, 2015.

TONINI, H.; BALDONI, A. B.; HOOGERHEIDE, E. S. S.; BOTELHO, S. D. C. C.. Caracterização e rentabilidade do sistema extrativista da castanha-do-brasil praticado em Itaúba (MT). Nativa, Sinop, v.5, n.3, p.175-181, 2017. DOI: http://doi.org/10.5935/2318-7670.v05n03a04

TONINI, H.; PEDROZO, C. A.. Variações anuais na produção de frutos e sementes de castanheira-do-Brasil (Bertholletia excelsa Bonpl., Lecythidaceae) em florestas nativas de Roraima. Revista Árvore, Viçosa, v.38, n.1, 2014. DOI: http://doi.org/10.1590/S0100-67622014000100013

WADT, L. H. O.; KAINER, K. A.. Domestication and breeding of the Brazil nut tree. Domestication and breeding: Amazonian species, Viçosa, p.297-317, 2012.

ZUIDEMA, P. A.. Demografía y manejo del árbol de castaña (Bertholletia excelsa). Riberalta: 2003.

A CBPC - Companhia Brasileira de Produção Científica (CNPJ: 11.221.422/0001-03) detém os direitos materiais desta publicação. Os direitos referem-se à publicação do trabalho em qualquer parte do mundo, incluindo os direitos às renovações, expansões e disseminações da contribuição, bem como outros direitos subsidiários. Todos os trabalhos publicados eletronicamente poderão posteriormente ser publicados em coletâneas impressas sob coordenação da Sustenere Publishing, da Companhia Brasileira de Produção Científica e seus parceiros autorizados. Os (as) autores (as) preservam os direitos autorais, mas não têm permissão para a publicação da contribuição em outro meio, impresso ou digital, em português ou em tradução. 